\title{
IDENTIFICAÇÃO DE SISTEMAS UTILIZANDO MÉTODOS POR SUBESPAÇOS
}

\author{
Santos Demetrio Miranda Borjas*, Francisco Magno Monteiro Sobrinho ${ }^{\dagger}$ \\ * Dept. DCEN, Universidade Federal Rural do Semi-Árido \\ Avenida Francisco Mota 570, CEP: 59625-900, \\ Mossoró,Rio Grande do Norte, Brasil
}

Emails: santos.borjas@ufersa.edu.br, magnomonteiro7@gmail.com

\begin{abstract}
This paper presents an overview of the problems of identification subspaces open loop. There are several algorithms that solve this problem, in which we can mention: MOESP, N4SID, IV-4SID, CVA. Based on the methods and MOESP N4SID MNS1 method is presented to identify deterministic systems operating in open loop. A simulated process is used to show the performance of this algorithm. The identification data is made to no noise, noise data with low intensity and high intensity noise.
\end{abstract}

Keywords - Identification; Subspace Methods; System identification; Multivariable Systems

Resumo - Neste artigo é apresentado uma visão geral sobre os problemas de identificação por subespaços em malha aberta. Existem diversos algoritmos que solucionam este problema, nos quais pode-se citar: MOESP, N4SID, IV-4SID, CVA. Baseados nos métodos MOESP e N4SID é apresentado o método MNS1 para identificar sistemas deterministicos operando em malha aberta. Um processo simulado é usado para mostrar o desempenho deste algoritmo. A identificação é feita para dados sem ruído, dados com ruídos de baixa intensidade e ruídos de alta intensidade.

Keywords- Identificação; Métodos por Subespaços; Sistema de identificação; Sistemas multivariaveis

\section{INTRODUÇÃO}

Existem métodos de identificação como "Métodos de Predição do Erro"(Prediction Error Methods - PEM) e os Métodos das Variáveis Instrumentais (Instrumental Variable Methods IVM), que são bastantes populares [10]. Recentemente, os métodos de identificação por subespaços têm emergido são como uma alternativa para os métodos tradicionais [2], [7], [8], [11], [12], [15], [16] , [17] e [19].

Nos métodos de identificação de sistemas dinâmicos por subespaços são tratados modelos de sistemas lineares invariantes no tempo em espaço de estados operando em tempo discreto. Pelas restrições citadas, pode parecer uma classe altamente restrita de modelos (especialmente por serem lineares), no entanto é bastante surpreendente como muitos processos industriais podem ser descritos com precisão por este tipo de modelo. Por outro lado, existe um grande número de ferramentas disponíveis de projeto de controladores para tais sistemas e modelos. Para empregar tais métodos é necessário o uso de ferramentas de teoria de sistemas, geometria e álgebra linear [4], [5],[6] e [16].

Os algoritmos de identificação por subespaços sempre consistem em dois passos. $O$ primeiro passo toma a projeção de certos subespaços gerados a partir dos dados, para encontrar uma estimativa da matriz de observabilidade estendida e/ou uma estimativa dos estados do sistema desconhecido. O segundo passo é recuperar as matrizes do sistema a partir da matriz de observabilidade estendida ou das estimativas dos estados.

Uma das ideias importantes ao se desenvolver a identificação por subespaços foi a reintrodução do conceito dos estados de um sistema dinâmico dentro do sistema de identificação. Em contraste com os algoritmos de identificação "clássicos", muitos dos algoritmos por subespaços primeiro estimam ou calculam os estados (implícita ou explicitamente), em seguida o modelo em espaço de estados é determinado. Esta é uma importante diferença entre os métodos clássicos, por exemplo PEM e os métodos por subespaços [3], [13] e [14].

\section{IDENTIFICAÇÃO POR SUBESPAÇO}

Os modelos por subespaços estão relacionados com sistemas e modelos da forma

$$
\begin{aligned}
x_{k+1} & =A x_{k}+B u_{k}+K e_{k} \\
y_{k} & =C x_{k}+D u_{k}+e_{k}
\end{aligned}
$$

Onde os vetores $u_{k} \in \Re^{m}$ e $y_{k} \in \Re^{l}$ são respectivamente, os valores medidos das entradas e saídas no instante $k$ dos processos com $m$ entradas e $l$ saída. O vetor $x_{k} \in \Re^{n}$ é o vetor de estados do processo em tempo discreto no instante $k$. $A, B$, $C, D$ são matrizes de dimensões apropriadas.

\subsection{PROBLEMA DE IDENTIFICAÇÃO}

Dado um conjunto de entradas $u_{k}$ e saídas $y_{k}$, determine a ordem $n$ do sistema desconhecido e as matrizes ( $A, B, C, D)[15]$.

\subsection{SOLUÇÃO IDEAL}

As equações (1) e (2) podem ser expressa na seguinte forma matricial [13]: 


$$
\left[\begin{array}{c}
X_{k+1} \\
Y_{k}
\end{array}\right]=\Theta\left[\begin{array}{l}
X_{k} \\
U_{k}
\end{array}\right]
$$

Onde

$$
\Theta=\left[\begin{array}{ll}
A & B \\
C & D
\end{array}\right]
$$

Corresponde aos parâmetros desconhecidos. A equação (3) pode ser interpretada como um modelo de regressão. Se na equação (3) as matrizes $X_{k+1}, Y_{k}, X_{k}$ e $U_{k}$ são dadas, então o parâmetro desconhecido $\Theta$ pode ser calculado pelo método dos mínimos quadrados, esto é:

$\hat{\theta}=\left[\begin{array}{cc}\hat{A} & \hat{B} \\ \hat{C} & \hat{D}\end{array}\right]=\operatorname{Min}=\left\|\left[\begin{array}{c}X_{k+1} \\ Y_{k}\end{array}\right]-\theta\left[\begin{array}{c}X_{k+1} \\ Y_{k}\end{array}\right]\right\|_{F}^{2}$

Onde $\hat{\theta}$ denota a estimativa de $\theta$ e $\|. .\|_{F}^{2}$ denota a norma de Frobenius de uma matriz. Da equação (4), resulta:

$\hat{\theta}=\left[\begin{array}{c}X_{k+1} \\ Y_{k}\end{array}\right]\left[\begin{array}{c}X_{k} \\ U_{k}\end{array}\right]^{T}\left(\left[\begin{array}{c}X_{k+1} \\ Y_{k}\end{array}\right]\left[\begin{array}{c}X_{k+1} \\ Y_{k}\end{array}\right]^{T}\right)^{-1}$

Então em um caso ideal, quando se têm os dados de entrada, saída e a sequência de estados para dois instantes de tempo sucessivos $k$ e $k+1$, a identificação do parâmetro $\Theta$ na equação (3) é trivial. No entanto na prática, $X_{k+1}$ e $X_{k}$ não são obtidos e têm que ser estimados dos dados de entrada e saída. Isto é um ponto importante nos métodos de identificação por subespaços. A diferença entre estes métodos reside na forma de como obter a sequência de estados estimados.

\subsection{EQUACÕES MATRICIAIS POR SUBE- SPAÇO}

Fazendo iterações sucessivas na equação (1), obtemos a seguinte equação matricial:

$$
Y_{f}=\Gamma_{n} X_{f}+H U_{f}
$$

Onde o símbolo $f$ denota os dados futuros e $p$ os dados passados. $\Gamma_{n}$ é a matriz de observabilidade estendida e definida por:

$$
\Gamma_{n}=\left(C^{T}(C A)^{T} \ldots\left(C A^{n-1}\right)^{T}\right)^{T}
$$

A matriz $H$ é definida como em [15]. Os dados passados e futuros das matrizes em bloco de Hankel são definidos por:

$$
U_{p}=\left[\begin{array}{ccc}
u_{0} & \cdots & u_{j-1} \\
\vdots & \ddots & \vdots \\
u_{i-1} & \cdots & u_{i+j-2}
\end{array}\right]
$$

De forma similar são definidas as outras matrizes $U_{f}, Y_{p}, Y_{f}$ e $W_{p}$. A sequência de estados $X_{k}$, pode ser computada usando projeção ortogonal ou projeção oblíqua para maiores detalhes ver $[8],[9],[16]$ e $[17]$.

\section{MÉTODOS DE IDENTIFICAÇÃO}

\subsection{MÉTODO MOESP}

O método MOESP soluciona o problema na seção 2.1. Aplicando certa projeção ortogonal na equação (6), obtém-se uma aproximação da matriz observabilidade estendida $\Gamma_{n}$ a partir da qual computa-se as matrizes $A$ e $C$. Para computar as matrizes $B$ e $D$ ver [17].

\subsection{MÉTODO N4SID}

O método N4SID soluciona o problema da seção 2.1. Aplicando-se certa projeção oblíqua na equação (6), obtêm-se uma aproximação para a sequência de estados $X_{k+1}$ e $X_{k}$. As matrizes do sistema formado pelas equações (1) e (2) são aproximadas utilizando-se a equação (5). Para maiores detalhes ver [16].

\section{MÉtodo PROPOSTO}

Baseado na técnica MOESP e N4SID é apresentado o método MNS1 para solucionar o problema apresentado na seção 2.1. Este método usa a técnica MOESP para determinar a matriz de observabilidade estendida $\Gamma_{i}$ e a técnica do método N4SID é aplicado para computar as matrizes $(A$, $B, C, D)$ através da equação (5). Uma estimativa da sequência de estados totais é dada por [14]

$$
X=\left(\Gamma_{i}\right)^{*} L_{32} L_{22}^{*}\left[\begin{array}{ll}
L_{21} & L_{22}
\end{array}\right] W_{p}
$$

Logo uma estimativa das matrizes $X_{k+1}$ e $X_{k}$ é dada através de:

$$
\begin{array}{r}
X_{k}=\left[x_{k}, x_{k+1}, x_{k+2}, \ldots, x_{k+N-2}\right] \\
X_{k+1}=\left[x_{k+1}, x_{k+2}, x_{k+3}, \ldots, x_{k+N-1}\right]
\end{array}
$$

O método N4SID computa duas projeções obliquas para estimar as equações (8) e (9) respectivamente. O método MNS1 usa uma projeção obliqua para computar as equações (8) e (9).

\section{SIMULAÇÃO}

Apresenta-se um modelo para avaliar o algoritmo proposto e comparamos como outros algoritmos existentes na idenficação (PEM, MOESP e N4SID).

\subsection{MODELO EM ESPACO DE ESTADOS COM MATRIZES ALEATÓRIAS}

A função "drss"do Matlab 6.5 permite gerar um modelo $\mathrm{M}$, discreto em espaços de estados, com matrizes do sistema $(A, B, C, D)$ na forma aleatória. A ordem escolhida do modelo é 4, que é igual ao posto $(A)$. Gerou-se um sistema MIMO 
de duas entradas e duas saídas. As matrizes do sistema são dadas por:

$$
\begin{gathered}
A=\left[\begin{array}{cccc}
0.2075 & 0.6037 & 0.0467 & 0.3296 \\
0.0647 & -0.1354 & 0.8093 & 0.2532 \\
-0.6549 & 0.2657 & -0.1127 & 0.4057 \\
-0.2056 & -0.5368 & -0.0829 & 0.3546
\end{array}\right] \\
B=\left[\begin{array}{cc}
0.1542 & 0 \\
-0.3429 & -0.9224 \\
-0.4785 & 1.5295 \\
0.5955 & -0.0031
\end{array}\right] \\
C=\left[\begin{array}{ccc}
-0.2141 & -0.9223 \\
-2.4532 & 0.0617 & -0.2908
\end{array}\right] \\
D=\left[\begin{array}{cc}
0 & 2.1346 \\
-0.5508 & 0.5446
\end{array}\right]
\end{gathered}
$$

Para o modelo $M$ com sinal de entrada $u$, foram coletados 1000 dados, dos quais 700 foram aplicados para identificação e o restante para a validação. Os sinais pré-tratados (feito pelo comando "detrend"do Matlab) usados na identificação são mostrados na figura 1 e 2.
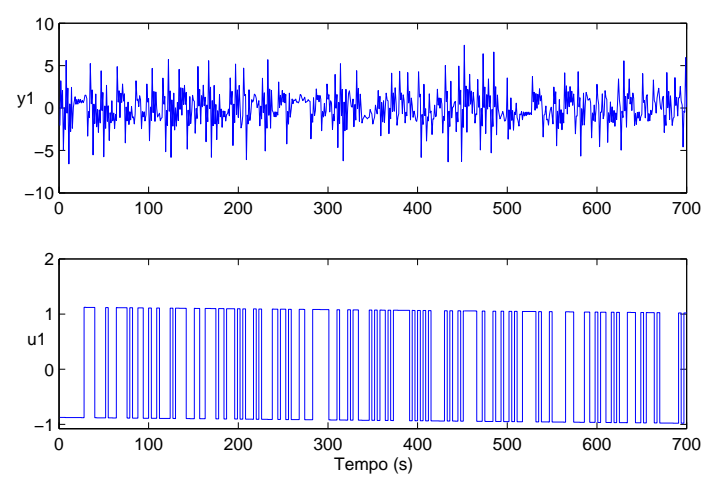

Figura 1: Sinal de saída $y_{1}$ e sinal de entrada $u_{1}$
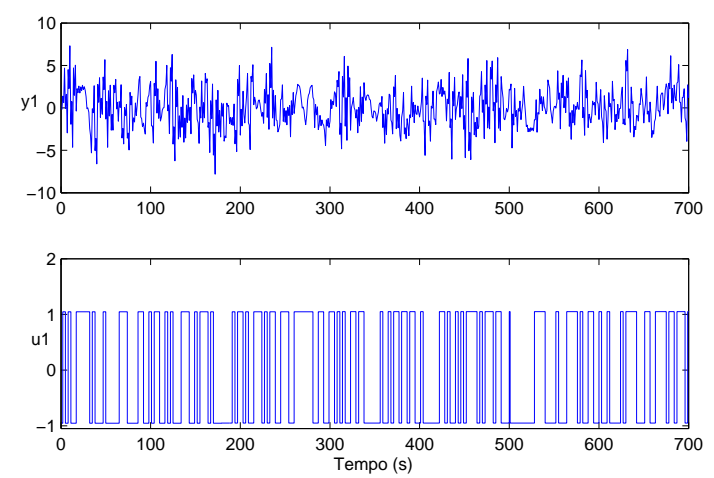

Figura 2: Sinal de saída $y_{2}$ e sinal de entrada $u_{2}$
O passo seguinte é encontrar o melhor modelo que simule o processo $M$, para os algoritmos citados anteriormente, mostrado na tabela 1. A ordem $n=6$ mostrada na figura 3 é dada pelos valores singulares mais significativos da matriz $S$, a qual é obtida da $S V D$ de certas projeções oblíquas ou ortogonais, dependendo do algoritmo sendo aplicado [16].

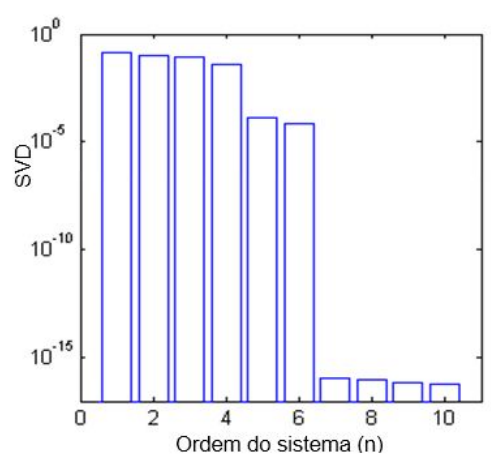

Figura 3: Valores singulares para o modelo $M$

Para avaliar a qualidade do modelo, aplicase indicadores de desempenho. Dentro dos quais existe a média da variância relativa $(M V A F)$ definido por:

$$
M V A F(\%)=\frac{1}{l} \cdot \sum_{i=1}^{N}\left(1-\frac{\operatorname{var}(y-\hat{y})}{\operatorname{var}(y)}\right) .100
$$

Onde $y$ é a saída real e $\hat{y}$ é a saída estimada pelo modelo obtido. O indice $M V A F$ é usado pelo $S M I$ toolbox. Este índice de desempenho é empregado para se avaliar a qualidade do modelo produzido por cada algoritmo, como mostra a tabela 1.

Tabela 1: Resultados numéricos do desempenho dos algoritmos

\begin{tabular}{lrc}
\hline Algoritmos & $\mathrm{T}(s)$ & Validação $(M V A F(\%))$ \\
\hline \hline MOESP & 0.297 & 100 \\
N4SID & 0.844 & 100 \\
MNS1 & 0.200 & 100 \\
PEM & 1.844 & 100 \\
\hline
\end{tabular}

Analisando os valores da tabela 1, todos os modelos tiveram um bom desempenho em termos de validação. Verifica-se que o tempo de processamento para obtenção do modelo é menor para $M N S 1$. Com o objetivo de visualizar o desempenho do algoritmo proposto, optou-se pelo método $M N S 1$ para identificar o processo.

A figura 4 mostra as saídas do processo real e aquelas geradas pelo modelo determinístico identificado (linha vermelha). Pode-se observar que o modelo identificado reproduz muito bem as principais características do processo. Foram consideradas condições iniciais nulas. 

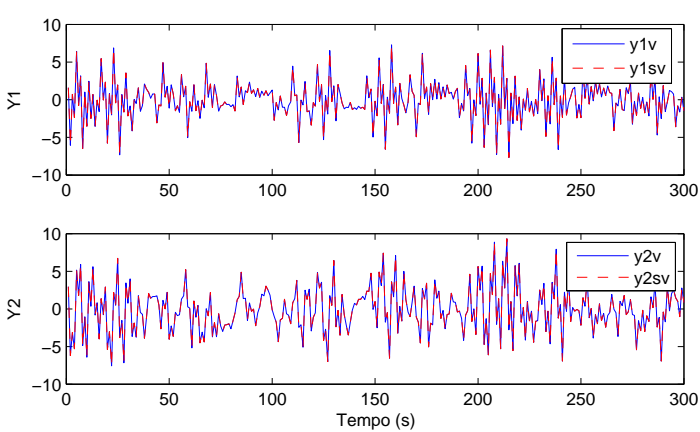

Figura 4: Comparação das respostas do processo real (linha azul) versus modelo (linha vermelha)

Se é conhecida a ordem do sistema real, esta não é necessário a mesma para o modelo obtido, portanto as matrizes do sistema real e do modelo obtido têm diferentes dimensões. As matrizes do modelo obtido são dadas por:

$A=\left[\begin{array}{cccccc}-0.845 & -0.372 & -0.048 & -0.180 & 0.042 & -0.114 \\ -0.235 & 0.463 & 0.783 & 0.269 & 0.091 & 0.032 \\ 0.039 & -0.752 & 0.364 & 0.444 & -0.110 & 0.010 \\ 0.052 & 0.067 & -0.011 & 0.332 & -0.109 & -0.336 \\ 0.000 & 0.000 & 0.000 & -0.000 & 1.001 & 0.008 \\ -0.000 & -0.000 & -0.000 & 0.000 & -0.000 & 0.998\end{array}\right]$

$$
B=\left[\begin{array}{cc}
-0.1814 & 3.2799 \\
0.1236 & 0.3198 \\
-0.0525 & -1.5857 \\
-1.0032 & 0.5198 \\
-0.0001 & 0.0001
\end{array}\right]
$$

$C=\left[\begin{array}{cccccc}-0.3505 & 0.2081 & 0.1006 & -0.3232 & -0.2805 & 0.040 \\ -0.3448 & 0.2530 & -0.4933 & 0.6984 & -0.0306 & 0.091\end{array}\right]$

$$
D=\left[\begin{array}{cc}
0 & 2.1346 \\
-0.5508 & 0.5446
\end{array}\right]
$$

A coleta de dados do processo real está sempre alterada por ruídos. Sistemas puramente determinísticos têm interesse apenas acadêmicos. Então se agregaram ruído de processo para os dados de entrada e saída. Neste caso, deseja-se saber como funciona o método de identificação por subespaços para o modelo dado na secção 4.

\subsection{IDENTIFICAÇÃO COM RUÍDO DE BAIXA (3\%) E ALTA (80\%) DE INTEN- SIDADE}

Sistemas com ruído na medição são mostrados na figura 5 [16].

Observe-se que o ruído não afeta a planta, somente afeta os sinais coletados de entradas e saídas. Os sinais de saída usados na validação para

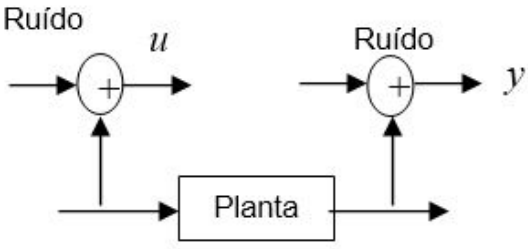

Figura 5: Sistema com ruído na medição

o sistema com ruídos de baixa (3\%) e alta (80\%) intensidade são mostrados nas figuras 6 e 7 e o efeito do ruído na saída são ilustrados nas figuras 8 e 9.

Quando o sistema esta perturbado por ruído, muitas vezes, encontrar a ordem $n$ do sistema a partir da decomposição de valores singulares não é fácil, uma alternativa é dada pelo critério de Akaike. Neste este caso $\mathrm{n}=4$ foi obtida pelo critério de Akaike [1] e [14]

Os resultados da simulação considerando os ruídos são mostrados na tabela 2 e 3 (considerando a ordem do sistema $\mathrm{n}=4$ ).
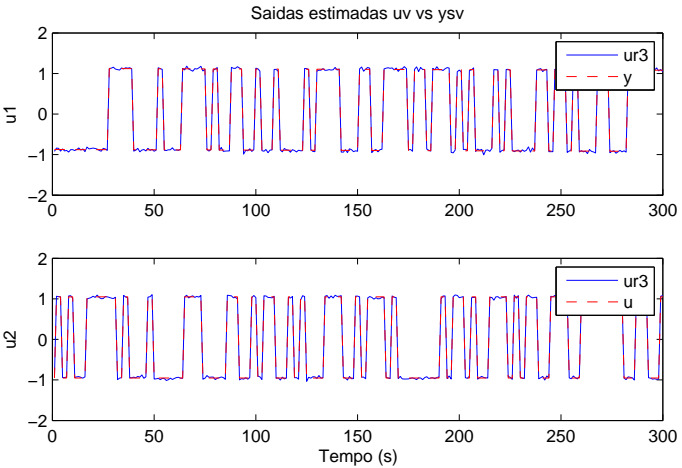

Figura 6: Dados de entrada com 3\% de ruído
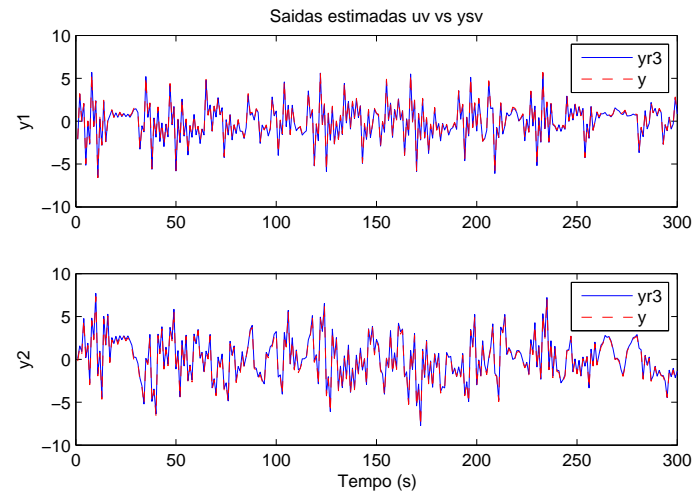

Figura 7: Dados de saída com 3\% de ruído

Analisando-se os valores da tabela 2, o modelo MNS1 é melhor em termos de validação. Verificase que o tempo de processamento é menor para o algoritmo MNS1.

Analisando-se os valores da tabela 3, o modelo MNSI é melhor em termos de validação, verifica- 

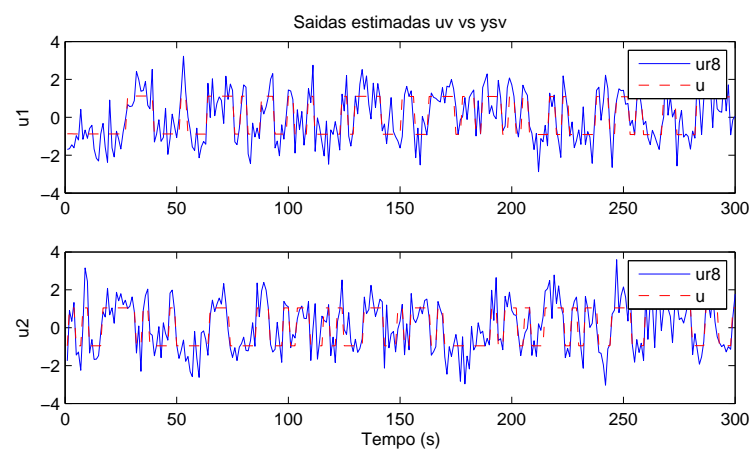

Figura 8: Dados de entrada com 80\% de ruído
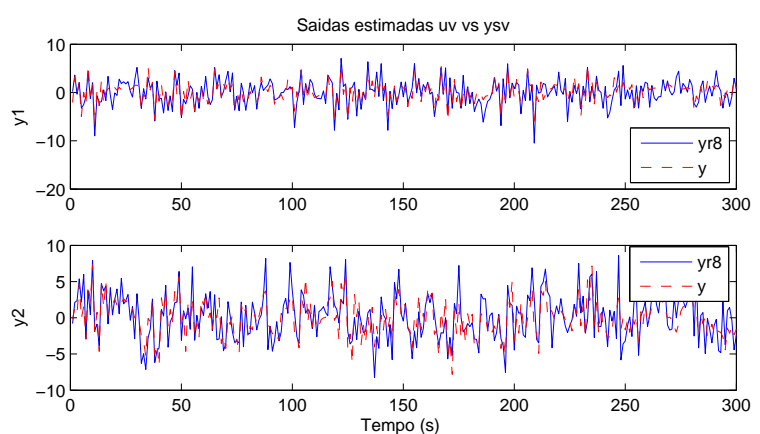

Figura 9: Dados de saída com $80 \%$ de ruído

Tabela 2: Resultados numéricos do desempenho dos algoritmos com ruídos de baixa intensidade $(3 \%)$

\begin{tabular}{lcc}
\hline Algoritmos & $\mathrm{T}(s)$ & Validação $(M V A F(\%))$ \\
\hline \hline MOESP & 0.281 & 99.78 \\
N4SID & 1.328 & 99.79 \\
MNS1 & 0.265 & 99.80 \\
PEM & 1.734 & 99.79 \\
\hline
\end{tabular}

Tabela 3: Resultados numéricos do desempenho dos algoritmos com ruídos de alta intensidade $(80 \%)$

\begin{tabular}{lcc}
\hline Algoritmos & T $(s)$ & Validação $(M V A F(\%))$ \\
\hline \hline MOESP & 0.203 & 38.15 \\
N4SID & 0.735 & 37.69 \\
MNS1 & 0.300 & 38.93 \\
PEM & 1.828 & 37.62 \\
\hline
\end{tabular}

se que o tempo de processamento para obtenção do modelo é menor para o algoritmo MOESP.

Dos resultados das tabelas 2 e 3 pode-se observar que os valores para todos os algoritmos são quase iguais, pois o ruído não ingressa no processo.

\section{CONCLUSÃO}

Neste artigo foi apresentado o método MNS1 para a identificação por subespaços em sistemas discretos invariante no tempo que trabalham em malha aberta. A matriz de observabilidade estendida é computada via técnica MOESP a partir desta é computada uma sequência de estados totais. A sequência de estados passados e futuros são computados desde a sequência de estados totais a qual tem unicamente um estado inicial, contrário ao método N4SID, pois para computar a sequência de estados passados e futuros usa-se duas projeções oblíquas diferentes, isto leva a um maior esforço computacional e muitas vezes gera um problema nas estimações.

Para avaliar o desempenho deste algoritmo, três algoritmos de identificação (MOESP, N4SID e PEM) foram aplicados a um processo mimo $M$ gerado pelo Matlab 6.5 e seus resultados foram comparados para dados sem ruídos, ruído com baixa intensidade e ruídos com alta intensidade. A comparação do desempenho destes algoritmos foi feita via validação cruzada, usando o critério MVAF. Para este caso especifico todos os algoritmos apresentaram bom desempenho. O modelo PEM foi o mais lento nos três casos de identificação

A identificação determinística é um caso ideal, pois na prática os dados encontra-se corrompidos por ruídos, no entanto o modelo MNS1 pode ser ampliado para a identificação de sistemas determinísticos - estocásticos.

\section{Agradecimentos}

- Agradecemos a FAPERN pelo apoio financeiro para a realização deste trabalho.

\section{Referências}

[1] Akaike, H. (1973), "Information theory and an extension of the maximum likelihood principle". In: Second International Symposium on Information Theory, Budapest, Hungary. Petrov, B.N.; Csaki, F.; (Eds.), pp. 267-281.

[2] Cott, B. (1995), "Introduction to the Process Identification", Workshop at the 1992 Canadian Chemical Engineering Conference, Journal of Process Control, Vol. 5, No. 2, pp. 67-69. DOI: 10.1016/0959-1524(95)90341-B

[3] Cock, K. (2003), and B. De Moor, "Subspace identification methods", in Contribution to section 5.5, Control systems robotics and automation of EOLSS, UNESCO Encyclopedia of Life Support Systems, (Unbehauen H.D.), Vol. 1 of 3, Eolss Publishers Co., Ltd. (Oxford, UK), pp. 933-979.

[4] Moor,B. De P.; Van Overschee and Favoreel, W. (1999), "Algorithms for subspace state space system identification - an overview". In B. Datta (Ed.), Applied and computational control, signal and circuits, Vol. 1, pp. 247311. Birkhauser: Boston (Chapter 6). 
[5] Favoreel, W. (2000), B. De Moor and P. Van Overschee, "Subspace state space system identification for industrial processes". Journal of Process Control, Vol. 10, No. 2-3, pp. 149-155. DOI: 10.1016/S0959-1524(99)00030-X

[6] Haverkamp, B. and Verhaegen, M. (1997), "SMI Toolbox: state space model identification software for multivariable dynamical systems", Vol 1, Delft University of Technology, The Netherlands.

[7] Katayama, T. (2005), "Subspace methods for system identification", Springer, London.

[8] Larimore, W. (1990), "Canonical variate analysis in identification, filtering and adaptive control". Proc. 29th Conference on Decision and Control, Hawai, USA, pp. 596-604. DOI: 10.1109/CDC.1990.203665

[9] Larimore, W. (1999), "Automated multivariable system identification and industrial applications". In: American Control Conference, ACC'99, San Diego, CA, Proceedings, Vol. 2, pp. 1148-1162.

[10] Ljung, L. (1999), "System Identification Theory for the User", Prentice Hall Englewood Cliffs, NJ.

[11] Mercère, G.; Bako, L. and S. L.(2008) "Propagator-based methods for recursive subspace model identification", Signal Processing, Vol 88, No. 3, pp. 468-491.

DOI: 10.1016/j.sigpro.2007.09.012

[12] Roberto, P.; Kurka G. ; Cambraia H. (2008), "Application of a multivariable input-output subspace identification technique in structural analysis", Journal of Sound and Vibration, Vol. 312, No. 3, pp. 461-47.

DOI: $10.1016 /$ j.jsv.2007.07.089

[13] Miranda, S. Borjas (2004). "Estudo de identificação por subespaços e aplicação a um processo industrial", Dissertação de Mestrado, Laboratório de Automação e Controle- Depto.De Engenharia de Telecomunicações e Controle- Escola Politécnica da Universidade de São Paulo.

[14] Miranda, S. Borjas (2009). "Estudo da identificação por subespaços em malha aberta e fechada e proposta de novos algoritmos, Dissertação de Doutorado, Laboratório de Automação e Controle- Depto.De Engenharia de Telecomunicações e Controle- Escola Politécnica da Universidade de São Paulo.

[15] Van Overschee, P. and De Moor B. (1995), "A unifying theorem for three subspace system identification algorithms". Automatica, Special Issue on Trends in System Identification, Vol. 31, No 12, pp. 1853-1864.

[16] Van Overschee, P. and De Moor, B. (1996), "Subspace identification for linear systems:
Theory, implementation, applications". Dordrecht: Kluwer Academic Publishers.

DOI: $10.1007 / 978-1-4613-0465-4$

[17] Verhaegen, M. (1994), "Identification of the deterministic part of MIMO state space models given in innovation form from input-output data", Automatica (Special issue on Statistical Signal Processing and Control), Vol. 30, No 1, pp. 61-74. DOI: 10.1016/0005-1098(94)90229-1

[18] Viberg, M. (2002), "Subspace-based statespace system identification". Circuits, Systems and Signal Processing, Vol. 21, No 1, pp. 2337. DOI: $10.1007 / \mathrm{BF} 01211649$

[19] Borjas, S. Garcia, C. (2011). "Subspace Identification Industrial Processos". TEMA. Tend`encia em matem 'atica aplicada e computacional, vol. 12, pp. 183-194. DOI: 10.5540/tema.2011.012.03.0183 